\title{
A retrospective analysis of the prevalence of imprinting disorders in Estonia from 1998 to 2016
}

\author{
Maria Yakoreva ${ }^{1,2} \cdot$ Tiina Kahre $^{1,2} \cdot$ Riina Žordania $^{1} \cdot$ Karit Reinson $^{1,2} \cdot$ Rita Teek $^{1} \cdot$ Vallo Tillmann $^{3,4}$. \\ Aleksandr Peet ${ }^{3,4} \cdot$ Eve Õiglane-Shlik, ${ }^{3,4}$ Sander Pajusalu $\mathbb{1}^{1,2,5} \cdot$ Ülle Murumets ${ }^{1} \cdot$ Mari-Anne Vals $^{1,2,4} \cdot$ Pille Mee $^{6}$. \\ Monica H. Wojcik ${ }^{7,8} \cdot$ Katrin Õunap $\mathbb{1 D}^{1,2,8}$
}

Received: 21 January 2019 / Revised: 31 March 2019 / Accepted: 28 May 2019 / Published online: 11 June 2019

(c) The Author(s), under exclusive licence to European Society of Human Genetics 2019

\begin{abstract}
Imprinting disorders (ImpDis) represent a small group of rare congenital diseases primarily affecting growth, development, and the hormonal and metabolic systems. The aim of present study was to identify the prevalence of the ImpDis in Estonia, to describe trends in the live birth prevalence of these disorders between 1998 and 2016, and to compare the results with previously published data. We retrospectively reviewed the records of all Estonian patients since 1998 with both molecularly and clinically diagnosed ImpDis. A prospective study was also conducted, in which all patients with clinical suspicion for an ImpDis were molecularly analyzed. Eighty-seven individuals with ImpDis were identified. Twenty-seven (31\%) of them had Prader-Willi syndrome (PWS), 15 (17\%) had Angelman syndrome (AS), 15 (17\%) had Silver-Russell syndrome (SRS), 12 (14\%) had Beckwith-Wiedemann syndrome (BWS), 10 (11\%) had pseudo- or pseudopseudohypoparathyroidism, four had central precocious puberty, two had Temple syndrome, one had transient neonatal diabetes mellitus, and one had myoclonusdystonia syndrome. One third of SRS and BWS cases fulfilled the diagnostic criteria for these disorders, but tested negative for genetic abnormalities. Seventy-six individuals were alive as of January 1, 2018, indicating the total prevalence of ImpDis in Estonia is 5.8/100,000 (95\% CI 4.6/100,000-7.2/100,000). The minimum live birth prevalence of all ImpDis in Estonia in 2004-2016 was 1/3,462, PWS 1/13,599, AS 1/27,198, BWS 1/21,154, SRS 1/15,866, and PHP/PPHP 1/27,198. Our results are only partially consistent with previously published data. The worldwide prevalence of SRS and GNAS-gene-related ImpDis is likely underestimated and may be at least three times higher than expected.
\end{abstract}

Katrin Õunap

katrin.ounap@kliinikum.ee

1 Department of Clinical Genetics, United Laboratories, Tartu University Hospital, Tartu, Estonia

2 Department of Clinical Genetics, Institute of Clinical Medicine, University of Tartu, Tartu, Estonia

3 Department of Paediatrics, Institute of Clinical Medicine, University of Tartu, Tartu, Estonia

4 Children's Clinic, Tartu University Hospital, Tartu, Estonia

5 Department of Genetics, Yale University School of Medicine, New Haven, CT, USA

6 United Laboratories, Tartu University Hospital, Tartu, Estonia

7 Divisions of Newborn Medicine and Genetics and Genomics, Boston Children's Hospital, Boston, MA 02115, USA

8 Broad Institute of MIT and Harvard, Cambridge, MA, USA

\section{Introduction}

The four classical and most common imprinting disorders (ImpDis)—Prader-Willi syndrome (PWS; OMIM \#176270), Angelman syndrome (AS; OMIM \#105830), Beckwith-Wiedemann syndrome (BWS; OMIM \#130650), and Silver-Russell syndrome (SRS; OMIM \#180860)were clinically described in the 1950-60s. The molecular etiology of these syndromes, however, remained unclear for the next few decades. More than eight new ImpDis have since been discovered. Although each ImpDis itself is a relatively rare disease, all ImpDis together constitute a notable proportion of hereditary genetic diseases.

The molecular etiology and clinical presentations of ImpDis are highly variable, making the diagnosis of these disorders difficult and sometimes challenging. It is assumed that a significant portion of ImpDis cases remain undiagnosed and therefore the exact prevalence of these disorders is not known. Understanding the epidemiology of these 
Table 1 Studies on the prevalence of ImpDis

\begin{tabular}{|c|c|c|c|c|}
\hline & Estimated character & Study population & Period & Data \\
\hline \multicolumn{5}{|l|}{ Prader-Willi syndrome } \\
\hline Burd et al. [13] & Population prevalence & 9-30 years old & & $1 / 16,062$ \\
\hline Åkefeldt et al. [14] & Population prevalence & $0-25$ years old & & $1 / 8,333$ \\
\hline \multirow[t]{2}{*}{ Ehara et al. [15] } & Birth incidence & & 1980-1989 & $1 / 15,060$ \\
\hline & Population prevalence & $0-15$ years old & & $1 / 17,482$ \\
\hline Stromme et al. [7] & Prevalence & Norway & $1980-1985$ & $1 / 10,000$ \\
\hline \multirow[t]{2}{*}{ Whittington et al. [16] } & Population prevalence & One UK health region, all ages & & $1 / 52,000$ \\
\hline & Birth incidence & & & $1 / 29,000$ \\
\hline Smith et al. [17] & Birth prevalence & Australia & 1998-2000 & $1 / 25,000$ \\
\hline \multirow[t]{2}{*}{ Vogels et al. [18] } & Population prevalence & Flandria, all ages & & $1 / 76,574$ \\
\hline & Birth incidence & & 1993-2000 & $1 / 26,676$ \\
\hline Thomson et al. [19] & Birth prevalence & Disability services commission & 1953-2003 & $1 / 29,500$ \\
\hline \multirow[t]{2}{*}{ Õiglane-Shlik et al. [9] } & Birth prevalence & Estonia & 2000-2004 & $1 / 30,439$ \\
\hline & Population prevalence & 0-20 years old, Estonia & & $1 / 30,606$ \\
\hline Lionti et al. [20] & Birth prevalence & Australia & $2003-2012$ & $1 / 15,830$ \\
\hline Richard-De Ceaurriz et al. [21] & Birth incidence & France & & $1 / 7,937$ \\
\hline Bar et al. [22] & Birth incidence & France & 2013 & $1 / 21,000$ \\
\hline \multicolumn{5}{|l|}{ Angelman syndrome } \\
\hline Clayton-Smith and Pembrey [2] & Incidence & Mainly referrals to genetic consultation, all ages & Unknown & $1 / 20,000$ \\
\hline Clayton-Smith [1] & Population prevalence & & & $1 / 62,000$ \\
\hline Kyllerman [3] & Prevalence rate & 6-13 years, epilepsy, intellectual disability & $1975-1986$ & $1 / 12,000$ \\
\hline Petersen et al. [4] & Prevalence rate & Neuropaediatric clinic & $1983-1991$ & $1 / 10,000$ \\
\hline Steffenburg et al. [5] & Prevalence & & & $1 / 12,000$ \\
\hline Buckley et al. [6] & Incidence & Institutionalized, severe intellectual disability & 1998 & $1 / 20,000$ \\
\hline Stromme et al. [7] & Prevalence & Norway & $1980-1985$ & $1 / 15,000$ \\
\hline Thomson et al. [8] & Birth prevalence & Western Australia & 1953-2003 & $1 / 40,000$ \\
\hline \multirow[t]{2}{*}{ Õiglane-Šlik et al. [9] } & Birth prevalence & Estonia & 2000-2004 & $1 / 52,181$ \\
\hline & Population prevalence & 0-20 years old, Estonia & & $1 / 56,112$ \\
\hline Mertz et al. [10] & Birth incidence & Denmark & & $1 / 24,580$ \\
\hline AlSalloum et al. [11] & Birth prevalence & Saudi Arabia & 2004-2005 & $1 / 45,682$ \\
\hline Luk et al. [12] & Birth incidence & Hong Kong China & 1995-2015 & $1 / 22,305$ \\
\hline \multicolumn{5}{|l|}{ Beckwith-Wiedemann syndrome } \\
\hline Thorburn et al. [23] & Birth prevalence & Jamaica & $1966-1968$ & $1 / 13,700$ \\
\hline Higurashi et al. [24] & Birth prevalence & Japan & $1972-1985$ & $1 / 14,430$ \\
\hline Higurashi et al. [25] & & & & $1 / 22,063$ \\
\hline Higurashi et al. [26] & & & & $1 / 27,472$ \\
\hline Wiedemann [27] & Birth prevalence & Germany & $1978-1991$ & $1 / 11,969$ \\
\hline Arroyo Carrera et al. [28] & Birth prevalence & Spain & 1976-1997 & $1 / 79,520$ \\
\hline Bianchi et al. [29] & Birth prevalence & Italy & 1992-1999 & $1 / 65,486$ \\
\hline Halliday et al. [30] & Birth prevalence & Australia & $1983-2003$ & $1 / 35,580$ \\
\hline Mussa et al. [31] & Birth prevalence & Italy & 1996-2009 & $1 / 10,569$ \\
\hline Barisic et al. [32] & Birth prevalence & 16 European countries & 1990-2015 & $1 / 26,000$ \\
\hline
\end{tabular}

disorders is valuable and helps to improve the diagnostic yield of ImpDis, to observe changes in the diagnostic yield over time, and to provide appropriate health care services. Previously, a limited number of studies have explored the prevalence, incidence and other epidemiological data related to ImpDis (Table 1) [1-32]. Almost all have focused on the epidemiology of PWS, AS, and BWS, and a minority of these studies have been performed during the last 10 years. 
The prevalence of PWS, AS, and BWS demonstrates variation of up to sevenfold between countries without clear explanation. To the best of our knowledge, there have been no studies that have looked the actual prevalence or incidence of SRS, with only theoretical estimates of the prevalence of SRS available [33].

Estonia is a small country with a total population of $1,319,133$ people, of whom 275,399 are $0-19$ years old (data of Statistics Estonia on January 1, 2018, www.stat.ee). There are two tertiary care children's hospitals in the country: in Tallinn (Tallinn Children's Hospital) for northern Estonia and in Tartu (Children's Clinic, Tartu University Hospital) for southern Estonia. The size of this country and nationalized healthcare system make it possible to perform an epidemiological study that covers the whole population and detects the maximum possible number of patients with ImpDis. The aim of this study was to identify the nationwide prevalence of the most common ImpDis in Estonia, to trends in the live birth prevalence of these disorders from 1998 to 2016, and to compare these results with previously published data.

\section{Material and methods}

\section{Study subjects}

The study was conducted in the Department of Clinical Genetics of Tartu University Hospital (including a branch in Tallinn), the only genetic referral centre in Estonia that provides comprehensive genetic counseling and molecular diagnostics of ImpDis for all patients living in Estonia. From 2014 onward, we conducted a retrospective and prospective study to find all cases of both clinically and molecularly diagnosed ImpDis among patients referred to a medical geneticist or other medical specialists.

We retrospectively reviewed the clinical and laboratory data of all Estonian patients with molecularly diagnosed ImpDis from 1998 to 2014. Prospective data are available from 2014 to 2018. Relevant information was obtained from the database of molecular diagnostics laboratory of the Department of Clinical Genetics. We created a registry of all molecularly confirmed ImpDis patients, including their personal data, laboratory findings, age at diagnosis, life/ death status, and inheritance.

All individuals from this group who met the clinical diagnostic criteria for BWS [34] and SRS [33] were clinically classified as BWS or SRS, respectively (regardless of genetic testing results).

Additionally, several lectures to raise awareness of the clinical presentation, diagnosis, and principles of supervision and treatment of ImpDis were given at various departments at our institution (including neurology, neonatology, endocrinology, and general pediatrics) and other hospitals. Information about this study and descriptions of ImpDis were repeatedly provided at national medical meetings in 2014-2018. Physicians were also given the opportunity to request free genetic testing for their patients with a clinical suspicion of ImpDis using a special sample submission form.

An additional search for patients with possible BWS was also conducted using our hospital electronic health record system. The clinical data of patients with ICD-10 codes Q87.3 (Congenital malformation syndromes involving early overgrowth, including BWS), Q38.2 (Macroglossia), C64 (Malignant neoplasm of kidney, except renal pelvis, only Wilms' tumor), C22.2 (Hepatoblastoma), Q79.2 (Exomphalos), and K42 (Umbilical hernia, only in the case of surgery) were thoroughly examined in search of other BWS symptoms. Persons who got $\geq 2$ points in the clinical scoring system of BWS [34] and in whom genetic testing for ImpDis was not performed were invited to the medical genetics clinic, and a molecular genetic analysis of BWS was provided for all interested patients.

All reported variants have been submitted to the Global Variome shared Leiden Open Variation Database at www. LOVD.nl/GNAS (patient IDs: 00229574-00229576), www.LOVD.nl/MKRN3 (patient ID: 00229577), www. LOVD.n1/UBE3A (patient ID: 00229578), and www. LOVD.nl/SGCE (patient ID 00229579).

\section{Methods}

\section{Methylation-specific polymer chain reaction (PCR)}

From 1998 to 2012 all patients with clinical suspicion of PWS or AS were tested using methylation-specific PCR. The analysis was performed with the primers SNRPNcommon, SNRPN-Mat, and SNRPN-Pat. PCR products were analyzed using $2.5 \%$ agarose gel electrophoresis. A detailed description of the method has been previously published [9].

\section{Cytogenetic and fluorescence in situ hybridization (FISH) analyses}

Patients with a positive methylation-specific PCR reaction were additionally studied using a FISH DNA probe for the PWS/AS region (D15S63, SNRPN/imprinting center) and a $15 \mathrm{q}$ telomere-specific control probe (154P1) (Cytocell Ltd., Oxfordshire, UK). Translocations involving chromosomes 14 and 15 were tested using standard GTG-banding of peripheral blood chromosomes and FISH analysis with probes specific for regions $14 \mathrm{cen}, 15 \mathrm{p} 11.2$, 15cen, 15q11.2-q12, 15q22, 15q13.1, and 15q26. At least 15 metaphases were usually investigated for each case per 
analysis. A more complete description of these methods has been published previously [9].

\section{Chromosomal microarray (CMA)}

CMA was introduced into clinical practise in Estonia in 2010 as a first-tier diagnostic genetic test for the patients with unclear developmental delay and/or congenital anomalies [35]. As a secondary test, CMA was performed in patients with a high suspicion of specific ImpDis in whom the first-tier genetic testing was negative or with a specific suspicion of copy number variation. The analysis was carried out using a 300,000-SNP HumanCytoSNP-12 v2.1 BeadChip (Illumina, Inc., San Diego, CA, USA) and analyzed using GenomeStudio software (Illumina, Inc.). The cnvPartition plugin (Illumina Inc.) for GenomeStudio was used to detect long contiguous stretches of homozygosity and isodisomic chromosomal regions, with the minimum region size set to $5 \mathrm{Mb}$.

\section{Uniparental disomy (UPD) analysis}

Until 2010, the test for UPD of chromosome 15 was performed using a PCR with fluorescently labeled oligonucleotides and the following set of microsatellites from outside of the PWS/AS critical region: D15S123, D15S153, D15S125, D15S131, D15S100, and D15S211 [9]. Since 2010 UPD analysis was, if needed, carried out by comparative analysis of the SNPs using the CMA (described above) results of the patient and at least one of his/her parents. The important advantage of this method is the ability to detect a UPD of any chromosome, to distinguish isodisomy from heterodisomy and precisely determine the location of a segmental UPD.

\section{Methylation-specific multiplex ligation-dependent probe amplification (MS-MLPA) analysis of PWS/AS, BWS/SRS, UPD $(6,7,14)$, and GNAS loci}

MS-MLPA analysis of a region 11p15 has been a golden standard in the molecular diagnostics of BWS and SRS in Estonia since 2010. Most of the patients with suspected BWS or SRS born in 1998-2017, in whom only methylation-specific single nucleotide primer extension (MS-SNuPE) analysis of chromosomal region 11p15 was performed and returned negative, were additionally tested for methylation defects and copy number variations in imprinted regions 6q24, 7p12, 7q32, and 14q32 (UPD(6, 7, 14) MS-MLPA test). The analysis was done using patients' DNA archived in the molecular diagnostics laboratory. Only patients without alternative molecular diagnoses were involved in the testing. The PWS/AS MSMLPA was implemented in the molecular diagnostics laboratory of Tartu University Hospital in 2012, UPD (6, $7,14)$ MS-MLPA in 2015, and GNAS locus MS-MLPA in 2017. These analyses were performed using SALSA ${ }^{\circledast}$ MSMLPA ${ }^{\oplus}$ probemixes ME030 BWS/SRS, ME028 PWS/AS, ME032 UPD7-UPD14, and ME031 GNAS (MRC-Holland, Amsterdam, the Netherlands) according to the manufacturer's instructions. PCR products were analyzed on capillary sequencer using the Genescan software (ABI 3130XL Genetic Analyzer; Applied Biosystems, Darmstadt, Germany). MLPA data analysis was performed with the Coffalyser software (MRC-Holland). A detailed description of the methods has been previously published [36] and is available also on the manufacturer's website (MRC-Holland, https://mlpa.com).

\section{MS-SNuPE assay}

The MS-SNuPE assay was performed in 18 patients with different growth disorders and dysmorphic features in suspicion of ImpDis. This assay is based on the ABI PRISM ${ }^{\oplus}$ SNaPshot ${ }^{\oplus}$ Multiplex Kit (Applied Biosystems). PCR products were analyzed on capillary sequencer using the GeneMapper $^{\circledR}$ software (AB 3130 Genetic Analyzer; Applied Biosystems). The MS-SNuPE assay allowed the simultaneous characterization of ten imprinted loci in five chromosomes (PLAGL1, 6q24; IGF2R, 6q25; GRB10, 7p12; MEST, 7q32; ICR1, ICR2, and IGF2P0 in 11p15.5; MEG3 and IG-DMR in 14q32; SNRPN, 15q11.2). A detailed description of the method has been published previously [37].

\section{CDKN1C gene sequencing}

Sequencing of CDKNIC was carried out in patients with clinical suspicion of BWS and in some patients with features of SRS in whom MS-MLPA and MS-SNuPE returned with normal results. Primers for the amplification of CDKN1C gene were designed with the help of the Primer 3 software (available from URL: http://bioinfo. ut.ee/primer3/). Both coding exons (exons 1 and 2 as numbered in NG_008022.1) of CDKNIC gene were amplified by PCR and sequenced directly using standard methods.

\section{Next generation sequencing (NGS) analysis}

The NGS analysis was performed using TruSight One (TSO) panel (Illumina Inc., San Diego, California) in patients with a clinical suspicion of ImpDis caused by a function-affecting variant in a specific gene, for example as in central precocious puberty (CPP; OMIM \#615346) [38] or in myoclonus-dystonia syndrome (MDS; OMIM \#604149) [39], or in cases of other diagnostic hypotheses. 
The TSO panel includes 125,396 probes aimed to capture 11,946,514-bp-targeted exon regions. These regions consist of 4,813 genes, which according to Online Mendelian Inheritance in Man database (OMIM, http://www.omim.org/), are associated with known genetic disorders or clinical phenotypes. A detailed description of the method has been published earlier [40]. A trio whole exome sequencing (WES) on an Illumina HiSeq platform and later data reanalysis by Genomics Platform at the Broad Institute of Harvard and MIT (Broad Institute, Cambridge, MA, USA) was performed in one patient. Confirmation and segregation of variants detected by TSO panel or WES was performed by Sanger sequencing in the proband and his/her parents.

\section{Statistical analysis}

The live birth prevalence of PWS, AS, BWS, SRS, and pseudo- or pseudopseudohypoparathyroidism (PHP/PPHP; OMIM \#103580, \#603233, \#612462, and \#612463) was calculated by dividing the number of live births in Estonia during the period 2004-2016 by the total number of patients with these ImpDis born within the same period. We excluded all cases born before 2004 and in last 2 years (2017 and 2018) from prevalence calculations as some of the patients born in these years may have had the diagnosis of ImpDis made at a later age. According to statistics of Estonia, there were 190,387 live births from 2004 to 2016. Live birth prevalence was estimated via the Generalized Linear Model Analysis using GENMOD procedure of the SAS system, Release 8.2 (SAS Institute, 1999). The distribution of the prevalence cases was assumed to be binomial, and the default logit link function was used. The only variable factor in the model was the observation year. The mean (expected) prevalence rate for a given year and a corresponding $95 \%$ confidence interval were predicted with the OUTPUT statement of the GENMOD procedure. Differences were considered statistically significant if the $p$ value was less than 0.05 . The analysis included individuals diagnosed with PWS, AS, BWS, SRS, and PHP/PPHP.

\section{Statement of ethics}

All procedures performed were in accordance with the ethical standards of the Tartu University Hospital and Research Ethics Committee of the University of Tartu (approval date 03/16/2015 (246/T-13), 12/19/2016 (265/ M16) and 11/20/2017 (275/M-11_4)). Written informed consent was obtained from the eligible patients, parents or legal guardians of the children.

\section{Results}

From 1998 to the end of the study period, a total of 984 patients were tested for ImpDis in Estonia. A total of 656 patients were tested for PWS/AS, 233 for BWS/SRS, and 95 for other ImpDis. Genetic or epigenetic alterations were identified in $6.4 \%$ of all performed PWS/AS tests and in $7.7 \%$ of BWS/SRS tests. Altogether, $7.9 \%$ of all tested patients were diagnosed with molecularly confirmed ImpDis.

Eighty-seven individuals with ImpDis were identified: 27 (31\%) of them had PWS, 15 (17\%) AS, 15 (17\%) SRS, 12 (14\%) BWS, 10 (11\%) PHP or PPHP, 4 (5\%) CPP, 2 (2\%) Temple syndrome (TS14; OMIM \#616222), 1 (1\%) transient neonatal diabetes mellitus (TNDM; OMIM \#601410), and $1(1 \%)$ MDS. No cases of Kagami-Ogata syndrome (KOS; OMIM \#608149), maternal uniparental disomy of chromosome 20 (UPD(20)mat; OMIM \#617352), or other rare ImpDis have been found (Table 2).
Table 2 ImpDis diagnosed in Estonia since 1998 up to the end of this study

\begin{tabular}{llll}
\hline Imprinting disorder & Acronym & Number of patients & $\%$ of all ImpDis \\
\hline Prader-Willi syndrome & PWS & 27 & 31 \\
Angelman syndrome & AS & 15 & 17 \\
Silver-Russell syndrome & SRS & 15 & 17 \\
Beckwith-Wiedemann syndrome & BWS & 12 & 14 \\
Pseudo- and pseudopseudohypoparathyroidism & PHP/PPHP & 10 & 11.5 \\
Central precocious puberty & CPP & 4 & 4.5 \\
Temple syndrome & TS14 & 2 & 2 \\
Transient neonatal diabetes mellitus & TNDM & 1 & 1 \\
Myoclonus-dystonia syndrome & MDS & 1 & 1 \\
Kagami-Ogata syndrome & KOS & 0 & 0 \\
Maternal uniparental disomy of chromosome 20 & UPD(20)mat & 0 & 0 \\
Schaaf-Yang syndrome & SHFYNG & 0 & 0 \\
Birk-Barel syndrome & - & 0 & 0 \\
Total & & 87 & \\
\hline
\end{tabular}


The age at diagnosis varied from the prenatal period to 83 years of age. Prenatal diagnosis was made in one case of familial PHP and in one case of UPD-caused PWS. An 83year-old individual with BWS was identified due to segregation analysis of a familial duplication in 11p15.4-p15.5 region [41]. The mean age at which a molecular diagnosis of the most common and classic ImpDis was made decreased over time. In our case, the difference between the mean age of diagnosis of PWS for patients born in the periods 1998-2008 (1.31 years, range: 30 days to 8 years, $n=12$ ) and 2009-2018 (0.06 years or 22 days, range: prenatally to 30 days, $n=9$ ) was clinically important and notable, but not statistically significant, likely owing to the small sample size $(P=0.2641)$.

All patients with PWS, AS, PHP/PPHP, CPP, TS14, TNDM, and MDS had molecular confirmation of the diagnosis. In the case of SRS and BWS, both molecularly and clinically diagnosed cases were taken into account.

Out of 27 patients with PWS, deletion of a paternal chromosomal region 15q11-q13 was present in 11 (41\%), maternal UPD(15) in nine (33\%), and Robertsonian translocation involving chromosome 15 in one patient (4\%). The remaining six patients $(22 \%)$ had positive methylation-specific PCR test but tested negative by FISH and were assumed to have either maternal UPD(15) or other less common alterations. Unfortunately, parental DNA was not available for further investigations in these cases. The molecular cause of AS was a maternal 15q11-q13 deletion in 11 patients (73\%). Two (13\%) patients with AS had an isolated methylation defect in the region, one patient had paternal UPD(15) and one nonsense variant in the maternal copy of $U B E 3 A$ gene.

One third of all SRS (5/15) and BWS (4/12) patients fulfilled the clinical diagnostic criteria for these disorders $[33,34]$ but were negative for genetic abnormalities. Among the remaining ten molecularly confirmed SRS patients, hypomethylation of imprinting center 1 (IC1) was found in seven $(70 \%)$, UPD of chromosome 7 in two patients and duplication of maternal 11p15.4-p15.5 region in one patient. Hypomethylation of IC2 was found in three $(37.5 \%)$ BWS patients. The mosaic paternal UPD of chromosome 11 had the same frequency of $37.5 \%$ (3) and duplication of paternal 11p15.4-p15.5 region was found in $25 \%$ (2) BWS cases.

Out of a total ten PHP/PPHP patients, the molecular cause for five patients with PHP and three patients with PPHP was a missense variant in GNAS. The molecular cause of the two remaining PHP patients was abnormal methylation of DMRs in the GNAS complex and one of them was caused by a small heterozygous deletion in the STX16 gene (exons 5 and 6, exons numbered like in NG_011831.2). All four patients with CPP were the members of a single large family and had the same heterozygous paternally inherited variant c.326G $>$ A, p. (Cys109Tyr) in MKRN3 (RefSeq NM_005664.3). Both cases of TS14 had a concurrent trisomy-one patient with triple X syndrome and the other with mosaic trisomy 14 -in addition to maternal UPD(14) [42]. The only patient with TNDM had an atypical clinical presentation and isolated hypomethylation of the PLAGL1 and IGF2R genes [36]. The case of MDS was caused by a paternal heterozygous nonsense variant-c.21 G>A, p.(Trp7*)-SGCE (RefSeq NM_003919.2). All patients with PHP/PPHP, CPP, TS14, TNDM, MDS, and the most patients with SRS and BWS were diagnosed in the last 6 years due to improved diagnostic methods and increased physicians' awareness. The percentage of these rare ImpDis dramatically increased during the past few years (Fig. 1). Figure 1 illustrates the change of proportion of diagnosed ImpDis during 1998-2016, 1998-2017, and 1998-2018, respectively.

Seventy-six individuals were alive as of January 1, 2018, indicating the total prevalence of ImpDis in Estonia is 5.8/ 100,000 (95\% CI 4.6/100,000-7.2/100,000). The minimum

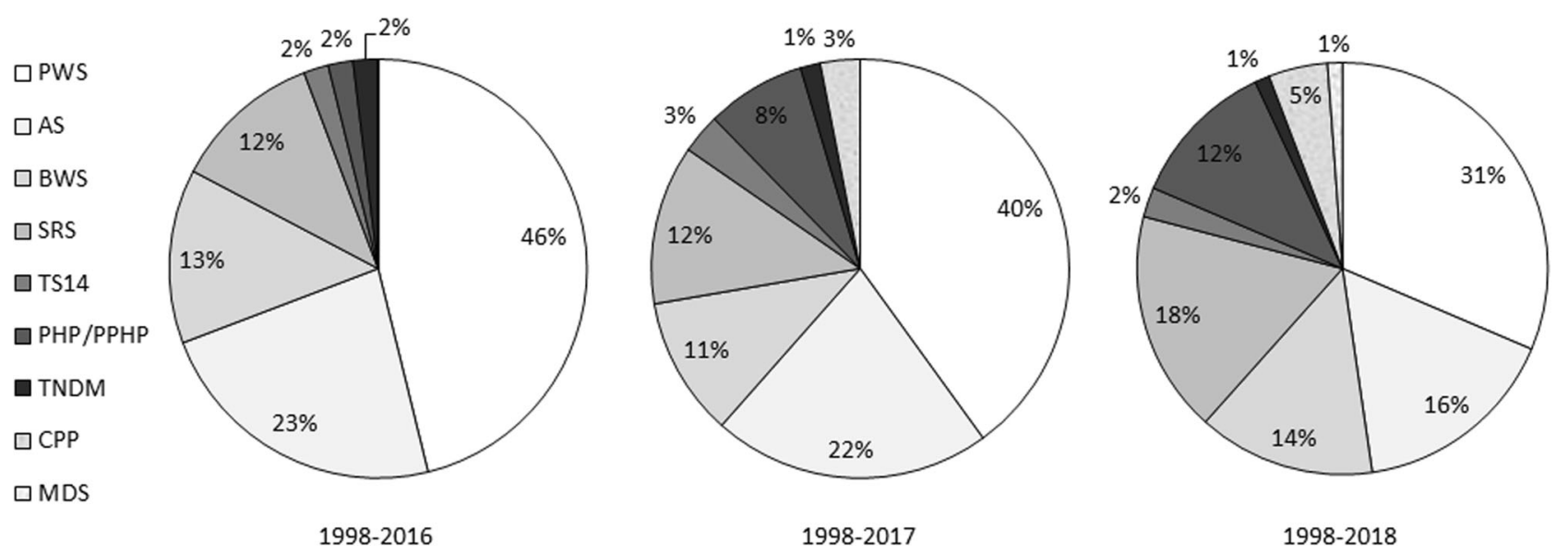

Fig. 1 Pie charts showing the percentage of all molecularly and clinically diagnosed ImpDis in Estonia during the periods 1998-2016, 1998-2017, and 1998-2018. Note the important increase in percentage of rare ImpDis (TS14, PHP/PPHP, TNDM, CPP, MDS) during the past few years 
A

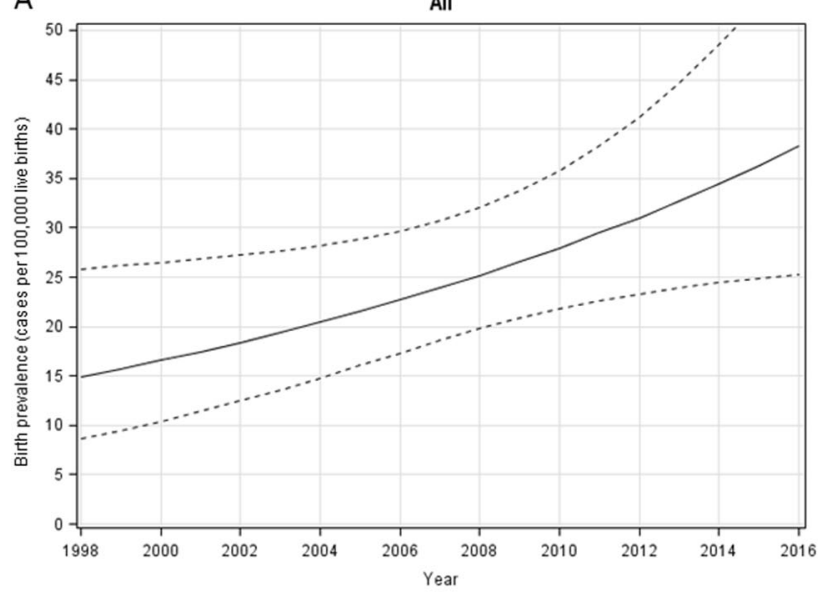

C

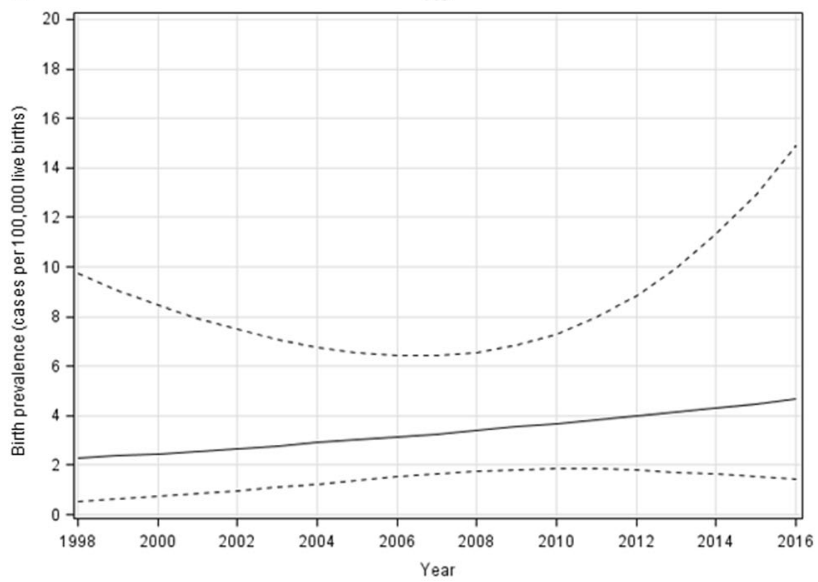

E

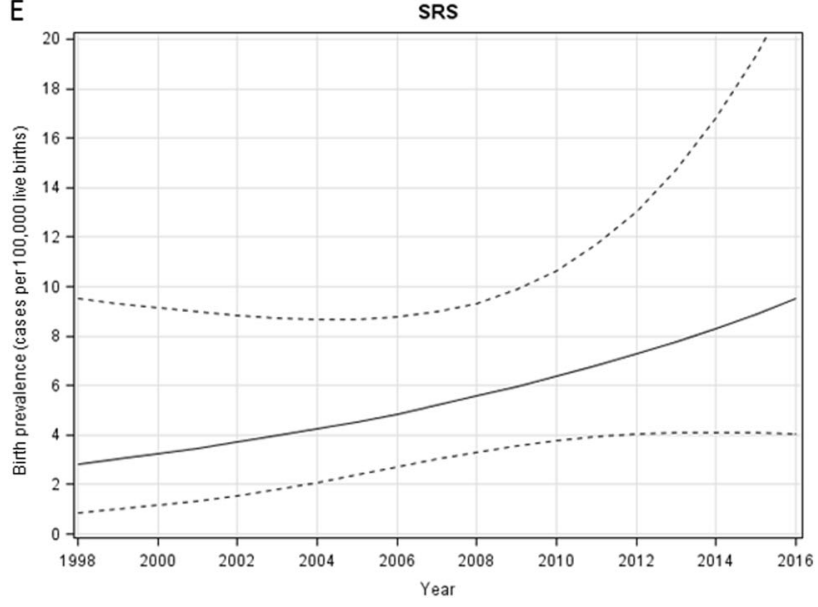

Fig. 2 Distribution of live birth prevalence of all ImpDis (a), PWS (b), AS (c), BWS (d), SRS (e), and PHP/PPHP (f) in Estonia from 1998 to 2016 by statistical logit analysis. The solid line represents birth prevalence and the dashed lines the 95\% confidence interval. Note a statistically significant increase in the live birth prevalence of all

live birth prevalence of all ImpDis in Estonia in 2004-2016 was $1 / 3,462$ (95\% CI 1/2,660-1/4,505) (Fig. 2a), PWS $1 /$ 13,599 (95\% CI 1/8,101-1/22,828) (Fig. 2b), AS 1/27,198
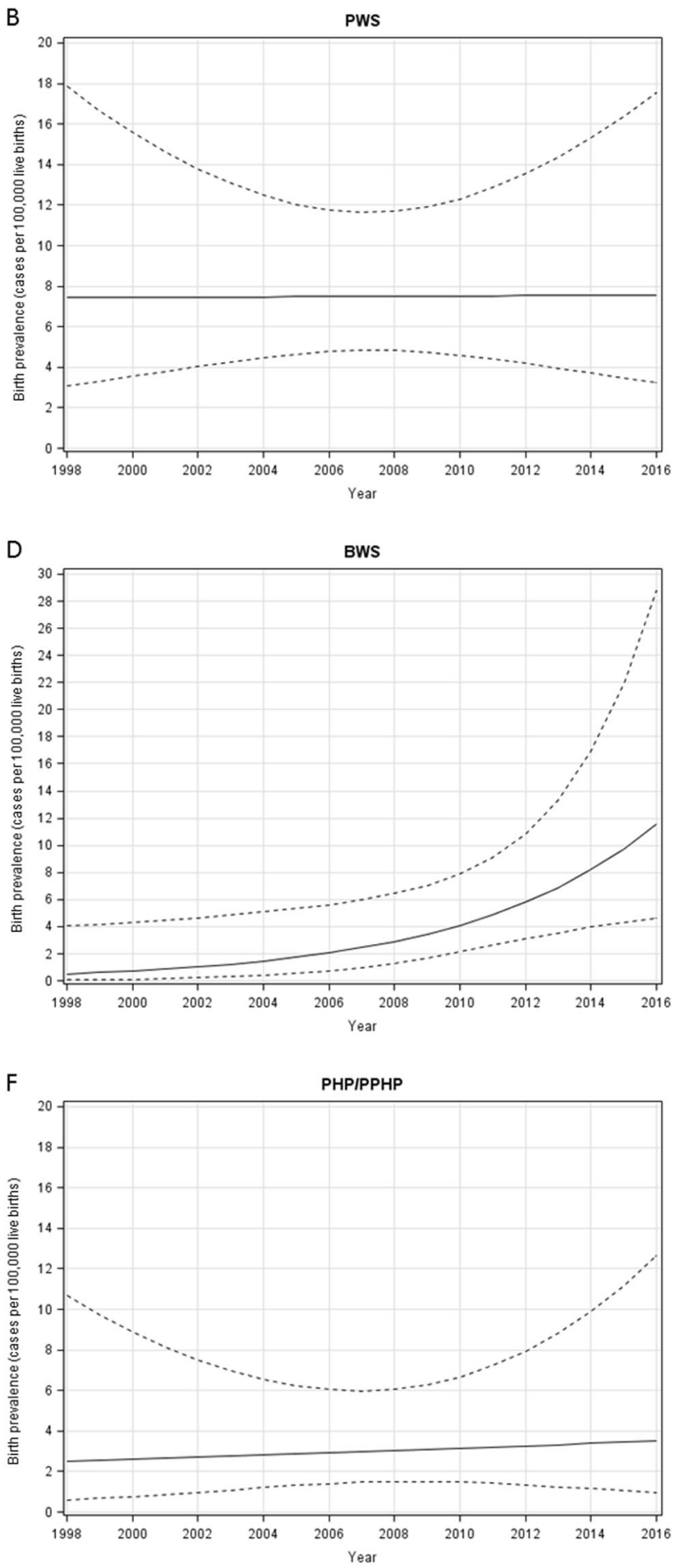

ImpDis $(P=0.027)$ and BWS $(P=0.0206)$. There was no statistically significant increase in the live birth prevalence of PWS $(P=0.9806)$, AS $(P=0.5331)$, SRS $(P=0.1851)$, and PHP/PPHP $(P=0.7792)$ during this period

(95\% CI 1/13,175-1/56,147) (Fig. 2c), BWS 1/21,154 (95\% CI 1/11,130-40,207) (Fig. 2d), SRS 1/15,866 (95\% CI 1/9,076-1/27,734) (Fig. 2e), and PHP/PPHP 1/27,198 
(95\% CI 1/13,175-1/56,147) (Fig. 2f). There was a statistically significant increase in the live birth prevalence of all ImpDis in Estonia during the years 1998-2016 (from 14.95/ 100,000 live births or $1 / 6,690$ in 1998 to $38.22 / 100,000$ or $1 / 2,616$ in 2016, $P=0.027$ ) (Fig. 2a). There was no statistically significant increase in the live birth prevalence of PWS (from 7.43/100,000 live births or 1/13,462 in 1998 to $7.57 / 100,000$ or $1 / 13,217$ in $2016, P=0.9806$ ) (Fig. $2 b$ ), AS (from 2.29/100,000 live births or $1 / 43,691$ in 1998 to $4.68 / 100,000$ or $1 / 21,386$ in $2016, P=0.5331$ ) (Fig. 2c), and PHP/PPHP (from 2.51/100,000 live births or 1/39,809 in 1998 to $3.52 / 100,000$ or $1 / 28,433$ in $2016, P=0.7792$ ) (Fig. 2f) during the last 19 years. However, there was a significant increase in the live birth prevalence of BWS (from $0.52 / 100,000$ live births or 1/92,456 in 1998 to $11.56 /$ 100,000 or $1 / 8,654$ in 2016, $P=0.0206$ ) (Fig. 2d) in Estonia during the years 1998-2016. The live birth prevalence of SRS in Estonia also increased during this period (from 2.84/100,000 live births or 1/35,157 in 1998 to 9.52/ 100,000 or $1 / 10,505$ in 2016), but this trend was not statistically significant $(P=0.1851)$ (Fig. 2e).

\section{Discussion}

We have determined the prevalence of all ImpDis in a single, nationwide population, an approach that has not been previously applied. It is thought that ImpDis are very rare disorders. However, we found the overall prevalence of ImpDis in Estonia to be $5.8 / 100,000(1 / 17,132)$. Compared to the prevalence of the most common metabolic disorders, such as mitochondrial disease $(1 / 5,000)$ [43] or phenylketonuria (1/ 10,000) [44], ImpDis are only 2-3 times less prevalent. Taking into account the birth prevalence of all ImpDis in Estonia in the last 13 years $(1 / 3,462$ during the period 2004-2016) it can be concluded that while each ImpDis, taken separately, is very rare, all ImpDis together are relatively common. Recently, a similar study of UPDs revealed that the incidence rate of all UPDs in the general population is also higher than expected at $1 / 4,000$ births [45].

The birth prevalence of PWS found in this study (1/ 13,599 ) is similar to that found by other studies (Table 1) and corresponds to an estimated worldwide prevalence of PWS 1/10,000-1/25,000 [46]. As seen in Table 1, the birth prevalence found in earlier publications is variable (1/ $7,937-1 / 30,439)[7,9,13-22]$ and our result is one of the highest. Compared to our previous 21-year study (1984-2004) of PWS in Estonia [9], the birth prevalence of PWS has more than doubled: from $1 / 30,439$ to $1 / 13,599$. However, at the end of the previous study (2004) a higher and very similar prevalence of PWS was observed (1/ 12,547) [9]. The statistical analysis also did not revealed any noticeable increase in the live birth prevalence of PWS in Estonia during the years 1998-2016. We can assume that the molecular diagnostic methods used in the last two decades allowed efficient detection of almost all patients with PWS and the true birth prevalence of the disorder in Estonia is approximately $1 / 13,000$ or 7.7/100,000. Previous studies have demonstrated that the mean age of molecular diagnosis of PWS significantly decreased during the last decade $[19,20]$. In our study we saw similar tendency. For those born between 1998 and 2008, the mean age of molecular diagnosis of PWS was 1.31 years compared to those who were born between 2009 and 2018 where the mean age was 0.06 years (22 days), but this increase was not statistically significant $(P=0.2641)$ due to small sample size.

We have also shown that the birth prevalence of AS in Estonia is $1 / 27,198$, which is lower than the estimated prevalence of 1/12,000-1/20,000 [46]. However, our results are in line with studies by Thomson et al. [8] and Mertz et al. [10] who found the prevalence of AS to be 1:40,000 in Western Australia and 1:24,580 in Denmark, respectively. Moreover, the obtained result is similar to that received at the end of the previous Estonian study $(1 / 23,640)$ [9], though the total AS birth prevalence for the whole period of 1984-2004 was almost two times lower $(1 / 52,181)$. Our statistical analysis showed an increase in the live birth prevalence of AS during the years 1998-2016. However, this trend was not statistically significant. This increase can likely be explained by the implementation of new diagnostic methods that allowed detection of methylation defects of region $15 \mathrm{q} 11$ q13, UPD(15) and UBE3A gene variants in addition to copy number anomalies. The birth prevalence of AS in Estonia is about $1 / 25,000$ or $4 / 100,000$, therefore, as it was shown in the previous Estonian survey [9], our study also does not confirm the general opinion that PWS and AS are equally represented disorders, as the live birth prevalence of AS was found to be 1.9 times less than that of PWS. The reason for this difference is not clear at present. Some studies have shown that paternal de novo variants and copy number anomalies are more prevalent than the maternal ones, and can likely be explained by high number of mitoses occurring in the sperm cells during male gametogenesis [47, 48]. This likely explains the higher prevalence of paternal de novo deletions of chromosomal region 15q11-q13 in the cases of PWS if compared to maternal deletions in AS.

The live birth prevalence of BWS revealed in this study $(1 / 21,154)$ is close to the prevalence of $1 / 26,000$ found in the latest population-based study of BWS covering a population of 16 European countries [32]. However, this is about 1.4 times lower than the expected prevalence of $1 /$ 15,000 [46]. The prevalence of BWS found in earlier studies varies greatly from $1 / 10,569$ [31] to $1 / 79,520$ [28] and our result falls in the middle of this range. As the prevalence of BWS was unexpectedly low at the beginning of our study, an advanced search for patients with clinical 
symptoms of BWS was performed. We assume that we have now likely identified the majority of BWS cases in Estonia and therefore the true live birth prevalence of BWS is $1 /$ 21,000 or $4.8 / 100,000$. We have also shown a significant increase in the live birth prevalence of BWS in Estonia from 1998-2016, most likely due to both improved diagnostic methods as well as increased physicians' awareness of BWS. Based on our previous experience with PWS, we can assume that the prevalence reached at the end of our study is close to the true prevalence of BWS in Estonia.

Surprisingly, we have found an unexpectedly high live birth prevalence of SRS in Estonia $(1 / 15,866)$, which differs greatly from the proposed SRS prevalence of $1 /$ 75,000-1/100,000 [46]. Even when considering only patients with molecularly confirmed SRS diagnosis, the prevalence is $1 / 27,198$ : about three times higher than estimated. To the best of our knowledge, there are no epidemiological studies on the prevalence of SRS and therefore our results are difficult to compare to other countries. We have shown an increase in the live birth prevalence of SRS in Estonia during the last 19 years, but this trend was not statistically significant. The cause of the high prevalence of SRS in Estonia is unclear. We propose that the true SRS prevalence is about $1 / 16,000$, which is higher than the prevalence of both BWS and AS.

The live birth prevalence of GNAS-gene-associated ImpDis (PHP and PPHP) found in our study was, similarly to SRS, unexpectedly high. Our prevalence of $1 / 27,198$ is ten times higher than the prevalence of 1/294,000 found in Japan [49] and more than three times higher than the prevalence of 1/90,900 in Denmark [50]. The prevalence of PHP and PPHP in Estonia is the same as the prevalence of AS. There was no statistically significant increase in the live birth prevalence of PHP/PPHP in Estonia during the years 1998-2016. As minimal data are available regarding the epidemiology of PHP/PPHP, and as our results may be influenced by the fact that most of our cases are familial (seven patients of nine are from two families), the exact prevalence of PHP/PPHP remains unknown. Though we assume that the true prevalence of PHP/PPHP is also higher than estimated. We did not calculate the prevalence of other less common ImpDis due to the small number of patients identified.

To the best of our knowledge, this is the first epidemiological study to estimate the birth prevalence of all ImpDis in a single population. We have demonstrated that the true prevalence of some ImpDis, like SRS and PHP/ PPHP, is significantly higher than previously estimated and that the live birth prevalence of all ImpDis together (1/ 3,462 ) can be compared with the prevalence of other relatively common genetic disorders. The significant increase in the diagnostic rate of some ImpDis in more recent years gives reason to suppose that the known prevalence of many ImpDis may further increase in the future.
Acknowledgements This work was supported by the Estonian Research Council grant PRG471, PUT355 and GARLA8175. Three authors (TK, KÕ, and MY) were members of the European Network of Congenital Imprinting Disorders (EUCID.net), which was supported by COST (BM1208). The Broad Center for Mendelian Genomics (UM1 HG008900) is funded by the National Human Genome Research Institute with supplemental funding provided by the National Heart, Lung, and Blood Institute under the Trans-Omics for Precision Medicine (TOPMed) program and the National Eye Institute. MHW is supported by T32GM007748. We thank the patients and their families for participating in this study.

\section{Compliance with ethical standards}

Conflict of interest The authors declare that they have no conflict of interest.

Publisher's note: Springer Nature remains neutral with regard to jurisdictional claims in published maps and institutional affiliations.

\section{References}

1. Clayton-Smith J. A clinical and genetic study of Angelman syndrome. Doctoral dissertation, Manchester University, 1993.

2. Clayton-Smith J, Pembrey ME. Angelman syndrome. J Med Genet. 1992;29:412-5.

3. Kyllerman M. On the prevalence of Angelman syndrome. Am J Med Genet. 1995;59:405. author reply 403-4.

4. Petersen MB, Brondum-Nielsen K, Hansen LK, Wulff K. Clinical, cytogenetic, and molecular diagnosis of Angelman syndrome: estimated prevalence rate in a Danish county. Am J Med Genet. 1995;60:261-2.

5. Steffenburg S, Gillberg CL, Steffenburg U, Kyllerman M. Autism in Angelman syndrome: a population-based study. Pediatr Neurol. 1996;14:131-6.

6. Buckley RH, Dinno N, Weber P. Angelman syndrome: are the estimates too low? Am J Med Genet. 1998;80:385-90.

7. Stromme P. Aetiology in severe and mild mental retardation: a population-based study of Norwegian children. Dev Med Child Neurol. 2000;42:76-86.

8. Thomson AK, Glasson EJ, Bittles AH. A long-term populationbased clinical and morbidity profile of Angelman syndrome in Western Australia: 1953-2003. Disabil Rehabil. 2006;28:299-305.

9. Oiglane-Shlik E, Talvik T, Zordania R, Poder H, Kahre T, Raukas E, et al. Prevalence of Angelman syndrome and Prader-Willi syndrome in Estonian children: sister syndromes not equally represented. Am J Med Genet Part A. 2006;140:1936-43.

10. Mertz LG, Christensen R, Vogel I, Hertz JM, Nielsen KB, Gronskov K, et al. Angelman syndrome in Denmark. birth incidence, genetic findings, and age at diagnosis. Am J Med Genet Part A. 2013;161A:2197-203.

11. AlSalloum A, El Mouzan MI, AlHerbish A, AlOmer A, Qurashi M. Prevalence of selected congenital anomalies in Saudi children: a community-based study. Ann Saudi Med. 2015;35:107-10.

12. Luk HM, Lo IF. Angelman syndrome in Hong Kong Chinese: a 20 years' experience. Eur J Med Genet. 2016;59:315-9.

13. Burd L, Vesely B, Martsolf J, Kerbeshian J. Prevalence study of Prader-Willi syndrome in North Dakota. Am J Med Genet. 1990;37:97-9.

14. Akefeldt A, Gillberg C, Larsson C. Prader-Willi syndrome in a Swedish rural county: epidemiological aspects. Dev Med Child Neurol. 1991;33:715-21.

15. Ehara H, Ohno K, Takeshita K. Frequency of the Prader-Willi syndrome in the San-in district, Japan.. Brain Dev. 1995;17:324-6. 
16. Whittington JE, Holland AJ, Webb T, Butler J, Clarke D, Boer H. Population prevalence and estimated birth incidence and mortality rate for people with Prader-Willi syndrome in one UK Health Region. J Med Genet. 2001;38:792-8.

17. Smith A, Egan J, Ridley G, Haan E, Montgomery P, Williams K, et al. Birth prevalence of Prader-Willi syndrome in Australia. Arch Dis Child. 2003;88:263-4.

18. Vogels A, Van Den Ende J, Keymolen K, Mortier G, Devriendt K, Legius E, et al. Minimum prevalence, birth incidence and cause of death for Prader-Willi syndrome in Flanders. Eur J Hum Genet. 2004;12:238-40.

19. Thomson AK, Glasson EJ, Bittles AH. A long-term populationbased clinical and morbidity review of Prader-Willi syndrome in Western Australia. J Intellect Disabil Res. 2006;50:69-78.

20. Lionti T, Reid SM, White SM, Rowell MM. A population-based profile of 160 australians with prader-willi syndrome: trends in diagnosis, birth prevalence and birth characteristics. Am J Med Genet A. 2015;167A:371-8.

21. Richard-De Ceaurriz B, Leymarie C, Godefroy A, Collignon P, Sigaudy S, Truc P. [Neonatal presentation of Prader-Willi syndrome: a report of five cases]. Arch Pediatr. 2017;24:1115-20.

22. Bar C, Diene G, Molinas C, Bieth E, Casper C, Tauber M. Early diagnosis and care is achieved but should be improved in infants with Prader-Willi syndrome. Orphanet J Rare Dis. 2017;12:118.

23. Thorburn MJ, Wright ES, Miller CG, Smith-Read EH. Exomphalos-macroglossia-gigantism syndrome in Jamaican infants. Am J Dis Child. 1970;119:316-21.

24. Higurashi M, Iijima K, Sugimoto $Y$, Ishikawa N, Hoshina $H$, Watanabe $\mathrm{N}$, et al. The birth prevalence of malformation syndromes in Tokyo infants: a survey of 14,430 newborn infants. Am J Med Genet. 1980;6:189-94.

25. Higurashi M, Iijima S, Takeshita T, Oda M, Takadaya K, Watanabe $\mathrm{N}$. Incidence of malformation syndromes and chromosomal abnormalities in 22,063 newborn infants in Tokyo. Jinrui idengaku zasshi. 1985;30:1-8.

26. Higurashi M, Oda M, Iijima K, Iijima S, Takeshita T, Watanabe $\mathrm{N}$, et al. Livebirth prevalence and follow-up of malformation syndromes in 27,472 newborns. Brain Dev. 1990;12:770-3.

27. Wiedemann HR. Frequency of Wiedemann-Beckwith syndrome in Germany; rate of hemihyperplasia and of tumours in affected children. Eur J Pediatr. 1997;156:251.

28. Arroyo Carrera I, Martinez-Frias ML, Egues Jimeno J, Garcia Martinez MJ, Eloina Cimadevilla Sanchez C, Bermejo Sanchez E. [Wiedemann-Beckwith syndrome: clinical and epidemiological analysis of a consecutive series of cases in Spain]. An Esp Pediatr. 1999;50:161-5.

29. Bianchi F. Registro nazionale malattie rare. Epidemiologia di 44 malformazioni congenite rare in Italia. 2002. http://www.iss.it/.

30. Halliday J, Oke K, Breheny S, Algar E, D JA. BeckwithWiedemann syndrome and IVF: a case-control study. Am J Hum Genet. 2004;75:526-8

31. Mussa A, Russo S, De Crescenzo A, Chiesa N, Molinatto C, Selicorni A, et al. Prevalence of Beckwith-Wiedemann syndrome in North West of Italy. Am J Med Genet Part A. 2013;161A:2481-6.

32. Barisic I, Boban L, Akhmedzhanova D, Bergman JEH, CaveroCarbonell C, Grinfelde I, et al. Beckwith Wiedemann syndrome: a population-based study on prevalence, prenatal diagnosis, associated anomalies and survival in Europe. Eur $\mathrm{J}$ Med Genet. 2018;61:499-507.

33. Wakeling EL, Brioude F, Lokulo-Sodipe O, O'Connell SM, Salem J, Bliek J, et al. Diagnosis and management of SilverRussell syndrome: first international consensus statement. Nature reviews. Endocrinology. 2017;13:105-24.
34. Brioude F, Kalish JM, Mussa A, Foster AC, Bliek J, Ferrero GB. et al.Expert consensus document: clinical and molecular diagnosis, screening and management of Beckwith-Wiedemann syndrome: an international consensus statement. Nat Rev Endocrinol. 2018;14:229-49.

35. Zilina O, Teek R, Tammur P, Kuuse K, Yakoreva M, Vaidla E, et al. Chromosomal microarray analysis as a first-tier clinical diagnostic test: Estonian experience. Mol Genet Genom Med. 2014;2:166-75.

36. Vals MA, Yakoreva M, Kahre T, Mee P, Muru K, Joost K, et al. The frequency of methylation abnormalities among Estonian patients selected by clinical diagnostic scoring systems for SilverRussell syndrome and Beckwith-Wiedemann syndrome. Genet Test Mol Biomark. 2015;19:684-91.

37. Begemann M, Leisten I, Soellner L, Zerres K, Eggermann T, Spengler S. Use of multilocus methylation-specific single nucleotide primer extension (MS-SNuPE) technology in diagnostic testing for human imprinted loci. Epigenetics. 2012;7:473-81.

38. Shin YL. An update on the genetic causes of central precocious puberty. Ann Pediatr Endocrinol Metab. 2016;21:66-9.

39. Rachad L, El Kadmiri N, Slassi I, El Otmani H, Nadifi S. Genetic aspects of Myoclonus-Dystonia syndrome (MDS). Mol Neurobiol. 2017;54:939-42.

40. Pajusalu S, Kahre T, Roomere H, Murumets U, Roht L, Simenson $\mathrm{K}$, et al. Large Gene Panel Sequencing in Clinical Diagnostics Results from 501 Consecutive Cases. Clin Genet. 2018;93:78-83.

41. Vals MA, Kahre T, Mee P, Muru K, Kallas E, Zilina O, et al. Familial 1.3-Mb 11p15.5p15.4 duplication in three generations causing Silver-Russell and Beckwith-Wiedemann syndromes. Mol Syndromol. 2015;6:147-51.

42. Yakoreva M, Kahre T, Pajusalu S, Ilisson P, Zilina O, Tillmann V, et al. A new case of a rare combination of temple syndrome and mosaic trisomy 14 and a literature review. Mol Syndromol. 2018;9:182-9.

43. Parikh S, Goldstein A, Karaa A, Koenig MK, Anselm I, BrunelGuitton C, et al. Patient care standards for primary mitochondrial disease: a consensus statement from the mitochondrial medicine society. Genet Med. 2017;19:1380.

44. van Wegberg AMJ, MacDonald A, Ahring K, Belanger-Quintana A, Blau N, Bosch AM, et al. The complete European guidelines on phenylketonuria: diagnosis and treatment. Orphanet J Rare Dis. 2017; 12:162.

45. Nakka P, McManus K, O'Donnell-Luria A, Francke U, Mountain $\mathrm{J}$, Ramachandran S, et al. Incidence of uniparental disomy in 2 million individuals from the $23 \mathrm{andMe}$ database. American society of human genetics meeting. San Diego, USA: 2018.

46. Eggermann T, Netchine I, Temple IK, Tumer Z, Monk D, Mackay $\mathrm{D}$, et al. Congenital imprinting disorders: EUCID.net-a network to decipher their aetiology and to improve the diagnostic and clinical care. Clin Epigenetics. 2015;7:23.

47. Acuna-Hidalgo R, Veltman JA, Hoischen A. New insights into the generation and role of de novo mutations in health and disease. Genome Biol. 2016;17:241.

48. Sibbons C, Morris JK, Crolla JA, Jacobs PA, Thomas NS. De novo deletions and duplications detected by array CGH: a study of parental origin in relation to mechanisms of formation and size of imbalance. Eur J Hum Genet. 2012;20:155-60.

49. Nakamura Y, Matsumoto T, Tamakoshi A, Kawamura T, Seino Y, Kasuga M, et al. Prevalence of idiopathic hypoparathyroidism and pseudohypoparathyroidism in Japan. J Epidemiol. 2000;10:29-33.

50. Underbjerg L, Sikjaer T, Mosekilde L, Rejnmark L. Pseudohypoparathyroidism-epidemiology, mortality and risk of complications. Clin Endocrinol. 2016;84:904-11. 